can be effective on public health threats in rural communities. Community-based preparedness and response should then take into account an integrated joint intervention package to mitigate public health threats.

Prehosp Disaster Med 2011;26(Suppl. 1):s69-s70

doi:10.1017/S1049023X11002378

\section{(A254) The Dutch Post-Disaster Response Strategy} E.F. Hall, ${ }^{1}$ J. Van Der Ree, ${ }^{1}$ C. Stom,${ }^{1}$ F. Greven ${ }^{2}$

1. IMG, Bilthoven, Netherlands

2. Groningen, Netherlands

The post-disaster response strategy in The Netherlands is unique in that it links scientific institutes, national government and local emergency response organizations. The lynch pin is the Centre for Environmental Health which was founded by the Ministry of Health to improve post-disaster care in The Netherlands. The recently refined Dutch strategy for post-disaster response will be presented and illustrated with a few examples from recent disasters. We will focus on both the role of the Centre and the role of public health Hazmat advisors who are part of the local emergency response organization. The latter advise on the health risks of exposure to CBRN agents. One of the main objectives of the Centre is to prepare guidelines and a structure to ensure transparent and authoritative advice is given to local governments and public health services on the need and value of post-disaster care. The Centre operates a front office, available $24 / 7$, to deliver integrated advice on public health and psychosocial care to local emergency response organizations. A network of experts with a wide range of expertise is on stand-by, whereby the characteristics of the disaster determine which experts compile the advice. The Centre also works closely with several other advisory organizations within the national emergency response organization. Three kinds of advice are delivered. Firstly, as an immediate response (usually within an hour), advice is given on the registration of victims. Secondly, usually within 24 hours, advice is given on the need and value of a health outcome assessment (HOA). Thirdly, if a HOA is decided on, detailed advice is given on its implementation. Another objective of the Centre is strengthening the unique position of regional public health services to deal with post-disaster care. The Centre produces guidelines, tools and training on demand to achieve harmonization and uniformity among these services.

Prehosp Disaster Med 2011;26(Suppl. 1):s70

doi:10.1017/S1049023X1100238X

\section{(A255) Medication Preparedness during Wildfire Evac-} uations

\section{Mayner, ${ }^{1}$ S.J. Stratton ${ }^{2}$}

1. School of Nursing and Midwifery, Adelaide, Australia

2. Center for Public Health And Disasters, Los Angeles, United States of America

Introduction: During wildfires, many are evacuated with little time to collect personal items. Evacuees who depend on daily medication for ongoing medical conditions often arrive to evacuation shelters without medication and with little knowledge of what they require. This problem is reported for evacuees in the 2008 Orange County California (USA) Freeway Triangle Wildfires.
Methods: Data was obtained retrospectively from Orange County Health Care Agency records regarding people who required medication while housed in evacuation shelters. Descriptive data was analysed using SPSS 17 and STATA 11.01.

Results: 40,000 persons were evacuated during the wildfires. Sixty of the evacuees aged from 6 to 82 years were without necessary medications. Of the sixty, there were 26 females and 34 males. People requiring medication would present to a public health nurse in the shelter whom would contact the Disaster Health Officer to arrange scripts for medication. Of the 60 people, $67 \%$ were unable to contact a primary physician and $75 \%$ were able to be issued a script for needed medication. The most common prescribed medication was albuterol for asthma and lung disease, then narcotic pain relief medication and next medication for cardiovascular / hypertension conditions.

Conclusions: Results show that life sustaining medication was required by people housed in an evacuation shelter. These people may not have had time to retrieve necessary medication if they had to evacuate quickly or may not have had an adequate supply of medication at the time of evacuation. Thus far there has been very little published on this issue however, our results show there is a need for pre-planning on behalf of people living in wildfire prone areas who require daily medication and are at risk for sudden evacuation. Our findings also highlight the important role provided by health workers in evacuation shelters in providing assistance for medication purposes.

Prehosp Disaster Med 2011;26(Suppl. 1):s70

doi:10.1017/S1049023X11002391

\section{(A256) Public Health: A Portal to Peace}

\section{G.V. Vroegindewey}

Center for Public and Corporate Veterinary Medicine, College Park, Maryland, United States of America

War, conflict, and complex emergencies are major contributors to the crisis of human suffering with impacts on health, public health infrastructure, food security, economic viability, community infrastructure, and social fabric as well as the environment. Conflict mitigation and resolution are essential to the recovery and restoration of the community and health. Public health can serve as a mechanism to mitigate the impacts of conflict, serve as a bridge to resolve conflict and provide community resilience. The role of health care professionals as a "Bridge to Peace" is a critical component of conflict resolution. Health as a Bridge for Peace was formally accepted by the 51st World Health Assembly in May 1998 as a feature of the "Health for All in the 21st Century" strategy and has been demonstrated across a wide range of conflicts. Public health has attributes that make it a valuable platform for conflict resolution: it is broad, populationbased, affects all parties, benefits both individuals and society, valued by recipients, and supports Universal Values. Public heath can be utilized in pre-conflict, conflict, and post-conflict situations and has been used in more than 20 conflict scenarios with Humanitarian Cease-fires, Days of Tranquility, and Safe/ Peace Corridors supporting programs such as childhood vaccination days in Afghanistan to Guinea Worm Eradication in East Africa.

Prehosp Disaster Med 2011;26(Suppl. 1):s70

doi:10.1017/S1049023X11002408 